\title{
Optical efficiency of Ag and Au nanoparticles
}

\author{
N.L. Dmitruk ${ }^{1}$, S.Z. Malynych ${ }^{1}$, I.E. Moroz ${ }^{2}$, V.Yu. Kurlyak ${ }^{3}$ \\ ${ }^{1} V$. Lashkaryov Institute of Semiconductors Physics, NAS of Ukraine, \\ 41, prospect Nauky, 03028 Kyiv, Ukraine \\ Phone: +38(044) 525-64-86, e-mail: s.malynych@gmail.com,dmitruk@isp.kiev.ua \\ ${ }^{2}$ National University "Lviv Polytechnica”, 12, S. Bandery str., 79013 Lviv, Ukraine \\ ${ }^{3}$ Ivan Franko Lviv National University, 8, Kyryla \& Methodiya str., 79005 Lviv, Ukraine
}

\begin{abstract}
In this paper, we present the results of calculations aimed at the optical radiation efficiency of $\mathrm{Ag}$ and $\mathrm{Au}$ nanoparticles, which is defined by the ratio of the scattering cross-section to the extinction one. The calculations were performed using Mie theory formalism for surrounding medium of various refractive indexes. It has been shown that silver nanoparticles exhibit substantially larger optical efficiency in a broad spectral range as compared to gold nanoparticles. The optical efficiency for silver nanoparticles with diameters over $90 \mathrm{~nm}$ exceeds $90 \%$.
\end{abstract}

Keywords: metal nanoparticles, optical properties, light scattering.

Manuscript received 02.06.10; accepted for publication 02.12.10; published online 30.12.10.

\section{Introduction}

Tendency towards miniaturization of various electronic devices as well as the development of experimental methods for manufacturing and characterization of nanoscale systems instigate growing interest to studying their physical properties. Metal nanoparticles, especially gold and silver are the most promising for practical applications. It is well known that visible light excites collective oscillations of free electrons inside metal particles, which are Localized Surface Plasmon Resonances (LSPR). In other words, LSPR are coupled oscillations of the electron density and electromagnetic waves. The excitation of LSPR in metal nanoparticles represents the most efficient process by which light interacts with matter and defines optical properties of nanoparticles. It is also very important that LSPR frequency could be successfully tuned within a broad spectral range by varying the refractive index of surrounding medium or by using the nanoparticles of different size and shape [1]. For gold and silver nanoparticles, the resonance falls into the visible region of electromagnetic spectrum. A striking consequence of this fact is bright colors exhibited by particles immersed into transparent matrix (e.g., aqueous suspensions or glass) both in transmitted and reflected light.

Up to date, it has been proposed a number of practical applications of gold and silver nanoparticles and nanoparticle arrays for needs of photonics [2], nonlinear spectroscopy [3], submicron visualization [4], surface-enhanced Raman and infrared spectroscopy $[5,6]$, photovoltaics [7], and various sensors [8]. The fundamental background of all the mentioned processes is a resonant enhancement of light interaction with metal nanoparticles, and it is crucial to minimize the losses caused by absorption of incident light, wasted as heat, by the metal nanoparticles for efficient energy transfer into the active materials. It is proposed to use the ratio of the scattering cross-section to the extinction cross-section as a quantitative parameter that describes efficiency of energy transfer [9]. This ratio or optical radiation efficiency represents the fraction of the energy reradiated from the particle out from the energy of the incident light.

\section{Localized surface resonances in metal nanoparticles}

As opposed to the surface plasmon-polaritons that are actually electromagnetic waves coupled to the electron plasma of a conductor and propagating along the metaldielectric interface plasmons in the subwavelength metal nanoparticles are localized collective oscillations of conducting electrons. The curved surface of the particle exerts an effective restoring force on the driven electrons, so that a resonance can arise, leading to field amplification both inside and in the near-field zone outside the particle. As a consequence, there is an effective scattering of incident light by metal nanoparticles just at the LSPR frequency. It also means 
that LSPR could be excited directly by incident light without any phase matching techniques, as it is required in the case of surface plasmon-polaritons.

Light extinction by small metal nanoparticles of the spherical shape can be described using simple quasistatic approximation provided that $R<<\lambda$. In that case polarizability defined as

$$
\alpha=4 \pi \varepsilon_{0} R^{3} \frac{\varepsilon-\varepsilon_{m}}{\varepsilon+2 \varepsilon_{m}}
$$

where $\varepsilon_{0}$ is the vacuum permittivity, $\alpha$ is the polarizability of particles, $R$ - radius of the particle, $\varepsilon$ and $\varepsilon_{m}$ are the permittivities of the particle and surrounding medium, respectively, $\lambda$ is the wavelength in vacuum. It is apparent that the polarizability experiences a resonant enhancement under the condition that the denominator is minimal, i.e. $\operatorname{Re}[\varepsilon(\omega)]=-2 \varepsilon_{m}$ (Fröhlich condition). Again, this expression is valid for very small $(R<<\lambda)$ particles. In this case, the phase of the harmonically oscillating electromagnetic field is practically constant over the particle volume, so that one can calculate the spatial field distribution by assuming the simplified problem of a particle in an electrostatic field. Then, the harmonic time dependence can be added to the solution, once the field distributions are known. This oscillation mode is exactly the dipole surface plasmon of metal nanoparticle. Tanabe [9] used quasistatic approximation to calculate the optical radiation efficiency of nanoparticles for a number of metals at fixed wavelengths.

However, for larger particles quasi-static approximation is no longer valid due to retardation effects, so a rigorous electrodynamic approach is required. Such a theory was firstly developed by G. Mie as early as in 1908. Nowadays, that theory is widely used and is treated in a variety of books [10]. According to the Mie theory, extinction, scattering, and absorption cross-sections can be calculated by series expansion of the internal and scattered fields into a set of partial waves described by vector harmonics:

$$
\begin{aligned}
& C_{\text {ext }}=\frac{2 \pi}{|k|^{2}} \sum_{L=1}^{\infty}(2 L+1) \operatorname{Re}\left(a_{L}+b_{L}\right), \\
& C_{s c a}=\frac{2 \pi}{|k|^{2}} \sum_{L=1}^{\infty}(2 L+1)\left(\left|a_{L}\right|^{2}+\left|b_{L}\right|^{2}\right), \\
& C_{a b s}=C_{\text {ext }}-C_{s c a}, \\
& \text { with }
\end{aligned}
$$

$$
\begin{aligned}
& a_{L}=\frac{m \psi_{L}(m x) \psi_{L}^{\prime} \psi^{\prime}(x)-\psi_{L}(x) \psi_{L}^{\prime}(m x)}{m \psi_{L}(m x) \xi_{L}^{\prime}-\xi_{L}(x) \psi_{L}^{\prime}(m x)} \\
& b_{L}=\frac{\psi_{L}(m x) \psi_{L}^{\prime}(x)-m \psi_{L}(x) \psi_{L}^{\prime}(m x)}{\psi_{L}(m x) \xi_{L}^{\prime}-m \xi_{L}(x) \psi_{L}^{\prime}(m x)}
\end{aligned}
$$

where $m=n / n_{m}, n$ and $n_{m}-$ are he complex refractive index of the nanoparticle and real refractive index of the surrounding medium, respectively, $\boldsymbol{k}$ is the wave vector, $x=|\boldsymbol{k}| R$ - size parameter, $\psi_{L}, \xi_{L}$ are Riccati-Bessel cylindrical functions. The prime indicates differentiation with respect to the argument. The summation index $L$ gives the order of the partial wave, $L=1$ corresponds to dipole fields, $L=2$ - to quadrupole, $L=3$ - to octupole fields, and so on.

Thus, by calculating the values of the extinction and scattering cross-sections one can obtain the optical radiation efficiency of nanoparticles:

$$
\eta_{\text {opt }}=\frac{C_{s c a}}{C_{e x t}}=\frac{C_{s c a}}{C_{s c a}+C_{a b s}} .
$$

\section{Results and discussion}

The calculations of the extinction and scattering crosssections by $\mathrm{Ag}$ and $\mathrm{Au}$ nanoparticles where performed using the modified version of Mie solution code [11]. For many practical purposes, especially in biophysics and sensorics, it is necessary to take into account the influence of surrounding medium on optical properties of nanoparticles. Fig. 1 depicts spectral dependences of the optical radiation efficiency $\eta_{\text {opt }}$ for spherical gold and silver nanoparticles of various diameters immersed into different surrounding media, namely: air, water, and fused silica. Note that optical efficiency of $\mathrm{Au}$ nanoparticles spreads over substantially narrower spectral range due to the strong interband transition at $\sim 2.4 \mathrm{eV}(515 \mathrm{~nm})$.

It is seen from Fig. 1 that the optical efficiency of $\mathrm{Au}$ and $\mathrm{Ag}$ nanoparticles has a trend to increase with increasing the particles' diameter. It occurred firstly due to increasing of the polarizability of larger particles, secondly as a consequence of fast scaling of the scattering cross-section $\left(\sim R^{6}\right)$ as compared to $R^{3}$ scaling of the absorption cross-section [12]. Calculations of the electric field distribution around the particle also confirm the size influence on the optical efficiency. In Fig. 2, the electric field distribution around $\mathrm{Ag}$ nanoparticles with the diameters 60 and $100 \mathrm{~nm}$ is shown. The curves correspond to the electric field distribution along $O y$ direction, while incident beam propagates along $z$ direction with polarization plane $x z$. One can see that for larger particles the electric field close to the particle's surface is more strong leading to the enhancement of the light-nanoparticle interaction. Thus, 100-nm silver nanoparticles act as more efficient scatterers. The calculations were performed employing field simulator freely available online [13].

Apparently, the size of the particles cannot be increased indefinitely where geometrical optics laws take place, while in Mie theory formalism the particles must be smaller than the optical wavelength. Quasi-static approximation also yields only rough values of the optical efficiency. From the calculations performed by Tanabe [9], it follows that the optical efficiency of silver nanoparticles in the visible spectral range exceeds $90 \%$, 

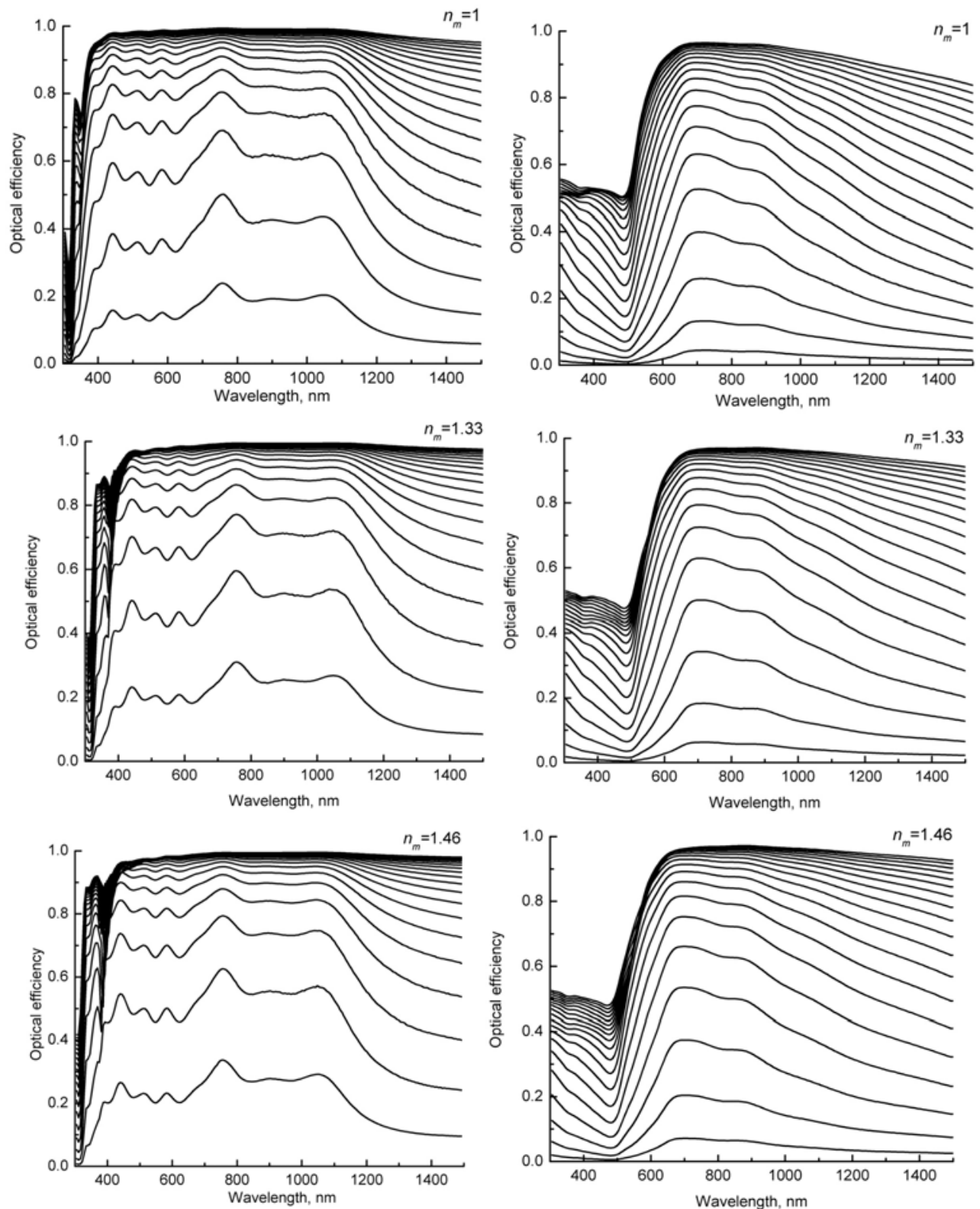

Fig. 1. Optical radiation efficiency of Ag (left-hand panel) and Au (right-hand panel) nanoparticles in air $\left(n_{m}=1\right)$, water $\left(n_{m}=1.33\right)$ and fused silica $\left(n_{m}=1.46\right)$. The lowest curves in all the plots correspond to the particles diameter of $20 \mathrm{~nm}$, while the highest ones to the diameter of $200 \mathrm{~nm}$ with the 10 -nm step.

when the particles' diameter reaches $150 \mathrm{~nm}$. This value is rather underestimated, since the corresponding diameter obtained from the Mie theory amounts $90 \mathrm{~nm}$. Fig. 3 depicts the diagram particle diameter - spectral range that represents the lines of the same optical efficiency of Ag and Au nanoparticles in air at 90 and $95 \%$.

Surrounding medium constitutes another important issue, which determines optical properties of the nanoparticles. Its influence was thoroughly studied in $[1,13]$. In particular, it has been shown that the extinction maxima in the wavelength scale red-shift linearly with increasing the refractive index of the medium [13]. In the cited papers, only spectral position of the extinction maxima with $n_{m}$ is discussed, while the absolute values of the maxima are not considered. It is established that extinction (scattering) cross-sections increase with increasing $n_{m}$.

Fig. 1 demonstrates that optical efficiency curves do not exhibit notable spectral changes in different surrounding media. Besides, the values of $\eta_{\text {opt }}$ increase with increasing the refractive index of surroundings. It can be explained from the fact that metals possess the negative permittivity. In that case, the optical contrast increases with increasing $n_{m}$ following by domination of scattering over the absorption. In a sense, surrounding 
medium amplifies the effect from metal nanoparticles. Augmentation of the optical efficiency due to increasing of $n_{m}$ is more notable for the particles with a diameter of 20-70 nm. For larger particles, the increase of the optical efficiency with the refractive index of surrounding medium is less pronounced.

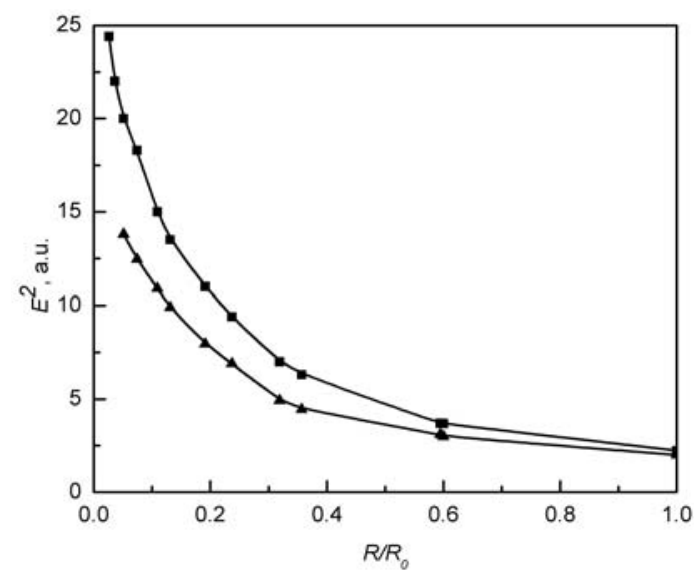

Fig. 2. Electric field distribution around 60-nm (A) and 100$\mathrm{nm}(\boldsymbol{\square})$ diameter silver nanoparticles embedded in air $\left(n_{m}=1\right)$. $R$ and $R_{0}$ denote a distance from the surface of the particle and radius of the particle, respectively.
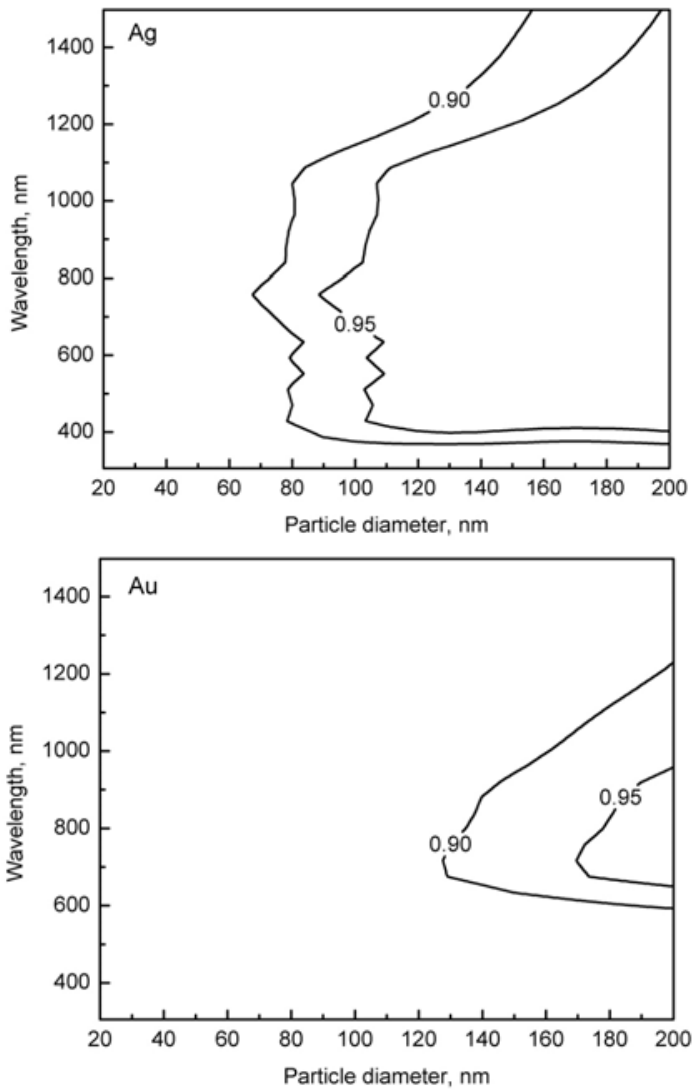

Fig. 3. The diagram diameter - spectral range representing the optical efficiency of 90-nm Ag and Au nanoparticles in air. The same intensity lines of the optical efficiency at the values of 0.9 and 0.95 are shown.

\section{Conclusions}

The optical radiation efficiency of metal nanoparticles or energy fraction of the incident light reradiated from the particle, but not wasted as heat due to absorption is one of the important characteristic of the nanoparticles. Using the Mie theory, we calculated the extinction and scattering cross-sections of gold and silver spherical nanoparticles of various diameters and obtained optical efficiencies defined by the ratio of the scattering crosssection to the extinction cross-section. The calculations were performed for different surrounding media, namely: air, water, and fused silica. Silver nanoparticles exhibit a larger optical efficiency in a broad spectral range than the gold ones of the same diameter. The optical efficiency for both metals has a trend to increase with growing the particles' size. For 90-nm silver nanoparticles, the optical efficiency exceeds $90 \%$ over the whole visible range. The optical efficiency of $\mathrm{Au}$ and Ag nanoparticles increases, when nanoparticles are embedded into the medium with higher refractive index. The obtained data might be useful for the selection of materials and sizes of nanoparticles for various photonics devices.

\section{Acknowledgments}

We thank Prof. G. Chumanov (Clemson University, USA) for collaboration and fruitful discussions.

\section{References}

1. K.L. Kelly, E. Coronado, L.L. Zhao, and G.C. Schatz, The optical properties of metal nanoparticles: The influence of size, shape, and dielectric environment // J. Phys. Chem. B 107, p. 668-677 (2003).

2. W.L. Barnes, A. Dereux, T.W. Ebbesen, Surface plasmon subwavelength optics // Nature 424, p. $824-830$ (2003).

3. H. Shen, B. Cheng, G. Lu, et al., Enhancement of optical nonlinearity in periodic gold nanoparticle arrays // Nanotechnology 17, p. $4274-4277$ (2006).

4. R.J. Blaikie, D.O.S. Melville, Imaging through planar silver lenses in the optical near field // J. Opt A: Pure Appl. Opt. 7, p. S176-S183 (2005).

5. W.E. Doering, S.M. Nie, Single-molecule and single-nanoparticle SERS: examining the roles of surface active sites and chemical enhancement // J Phys Chem B 106, p. 311-317 (2002).

6. T.R. Jensen, R.P. Van Duyne, S.A. Jonson, V.A. Maroni, Surface-enhanced infrared spectroscopy: a comparison of metal island films with discrete and nondiscrete surface plasmons // Appl. Spectrosc. 54, p. 371-377 (2000).

7. K.R. Catchpole, A. Polman, Plasmonic solar cells // Optics express 16(26), p. 21793-21800 (2008). 
8. S. Malynych, G. Chumanov, Coupled planar silver nanoparticle arrays as refractive index sensors // $\mathrm{J}$. Opt. A: Pure Appl. Opt. 8, p. S144-S147 (2006).

9. K. Tanabe, Optical radiation efficiencies of metal nanoparticles for optoelectronic applications // Materials Letters 61, p. $4573-4575$ (2007).

10. C.F. Bohren, D.R. Huffman, Absorption and Scattering of Light by Small Particles. John Wiley \& Sons, Inc., 1983, p. 530.

11. J. Camden, G.C. Schatz, Nanosphere Optics Lab // DOI: 10254/nanohub-r1309.2 (2006).
12. S.A. Maier, Localized Surface Plasmons, Chap. 5, in: Plasmonics: Fundamentals and Applications. Springer, 223, 2007, p. 65-88.

13. B. Tejerina; T. Takeshita; L. Ausman, G.C. Schatz, Nanosphere Optics Lab Field Simulator // DOI: 10254/nanohub-r3056.2 (2007).

14. T.R. Jensen, M.L. Duval, K.L. Kelly, A.A. Lazarides, G.C. Schatz, R.P. Van Duyne, Nanosphere lithography: Effect of the external dielectric medium on the surface plasmon resonance spectrum of a periodic array of silver nanoparticles // J. Phys. Chem. B 103, p. 98469853 (1999). 